\title{
Catherine Langlais : une femme de science qui s'est épanouie dans la direction d'équipes au sein d'une grande société industrielle
}

Catherine Langlais a été contactée par Michel Spiro, président de la Société Française de Physique, pour devenir vice-présidente de notre société savante. Elle s'est présentée le 2 décembre 2016 à l'ensemble des responsables de la SFP, lors du séminaire annuel de travail à Marne-la-Vallée. Véronique Pierron-Bohnes, présidente de la commission Femmes et Physique (F\&P), en a profité pour lui poser quelques questions.

F\&P : Catherine Langlais, vous êtes directrice adjointe de la R\&D de Saint-Gobain, où vous avez effectué toute votre carrière. Vous avez relevé de nombreux défis tout au long de votre parcours. À l'époque où vous avez fait vos études, il n'était pas courant que les femmes soient scientifiques. Comment vous êtes-vous tournée vers les sciences?

Catherine Langlais (CL) : Au lycée, j'étais autant intéressée par les lettres que par les sciences. Lorsque l'école d'ingénieurs française la plus prestigieuse, l'École polytechnique, s'est ouverte à l'entrée des femmes en 1972, Anne Chopinet y est rentrée major et m'a donné envie d'essayer à mon tour. Je pensais que je pourrais exercer mon métier dans les sciences en gardant la littérature pour mes loisirs, l'inverse étant plus difficile. L'année suivante, je suis entrée à l'École des mines de Nancy dont je suis ingénieure. Pour motiver les jeunes à faire des sciences, je crois vraiment à la force des modèles, comme Anne Chopinet pour moi. Aussi, lorsque j'ai occupé des postes à responsabilités, je me suis toujours efforcée d'être un exemple, de montrer aux chercheuses que les femmes pouvaient s'épanouir dans les sciences et y faire des carrières tout à fait similaires à celles des hommes, tout en ayant des enfants si elles le souhaitaient et une vie privée heureuse.

F\&P : Quel parcours vous a amenée à diriger la recherche sur les matériaux de Saint-Gobain ? Quels ont été vos modèles et vos mentors?

CL : Dès l'École des mines, je me suis intéressée aux énergies renouvelables. J'ai effectué mon stage de fin d'études sur des maisons solaires au laboratoire du CNRS d'Odeillo, dont le four solaire est doté de miroirs fabriqués par Saint-Gobain. J'ai passé ensuite une année à l'Université de Stanford en Californie pour préparer un Master en 'Mechanical engineering'. Quand je suis revenue en France, l'énergie solaire n'était pas assez développée pour me permettre d'y trouver un emploi et je me suis alors souvenue des miroirs performants de Saint-Gobain. J'ai donc postulé dans cette entreprise, attirée par leurs recherches dans l'isolation des bâtiments. J'ai ainsi été embauchée comme jeune chercheuse à Isover, filiale de Saint-Gobain. Mes premières recherches furent consacrées à la compréhension des transferts de chaleur et de masse au sein des isolants fibreux. Ces travaux, qui me conduisirent à mener plusieurs collaborations avec des laboratoires académiques français, spécialistes des milieux poreux, poursuivaient des objectifs industriels très concrets. Je découvris ainsi une des grandes satis- factions que peut procurer la recherche industrielle : avoir à résoudre des problèmes appliqués, en s'appuyant sur une compréhension scientifique très approfondie de ceux-ci.

Je suis restée vingt ans au centre de R\&D d'Isover en France. J'ai apprécié les différents postes qui m'y ont été proposés : responsable d'équipe, responsable de projets, responsable de département... J'ai suivi avec joie l'éducation de mes deux enfants pendant cette période. Mon mari a toujours partagé les tâches quotidiennes. Je n'aurais jamais eu cette carrière s'il ne m'avait pas épaulée et soutenue. De toutes ces années, j'ai retenu que la qualité du temps que vous passez avec votre famille est plus importante que sa quantité. Je me suis beaucoup investie dans mon travail, tout en apprenant à accepter que la perfection n'est pas toujours possible ! J'ai été ensuite nommée directrice du Centre de Recherche de Saint-Gobain à Aubervilliers où je suis restée onze ans, avant de prendre mes responsabilités actuelles. Pendant ces onze ans et grâce au travail de toutes les équipes, le périmètre du centre s'est élargi et ses activités, historiquement "verrières ", ont été étendues à toutes celles du groupe (en particulier plâtre, ciment et habitat en général). Dans ce contexte de croissance, l'effectif est passé de 270 à plus de 400 personnes et j'ai pu contribuer à l'internationalisation des équipes (la proportion de chercheurs étrangers passant de quelques pourcents à près de 23\%) et à leur féminisation (de 29 à 36\%).

\section{F\&P : Quelles sont vos responsabilités actuelles?}

CL : Depuis 2011, je suis plus spécialement chargée de la coordination du réseau des grands centres de recherche de Saint-Gobain (trois en Europe, un aux USA, un en Inde et un en Chine), afin d'offrir aux différents "business" un ensemble cohérent et efficace. Je coordonne également certains programmes de recherche dits "transversaux " autour de la science des matériaux.

De 2010 à 2013, j'ai été vice-présidente du Haut Conseil à la Science et à la Technologie, chargé d'éclairer le gouvernement sur toutes les questions relatives aux grandes orientations de la nation en matière de politique de recherche scientifique, de transfert de technologie et d'innovation. Sous la présidence de Jean Jouzel, nous avons remis plusieurs rapports qui n'ont pas toujours eu l'écho que nous aurions souhaité ! Je suis aussi depuis vingt ans membre du jury du prix Roberval, prix du livre et de la communication en technologie, ayant pour objectif de favoriser le développement d'une culture technologique francophone. Enfin, depuis 2013, je fais partie de l'Académie des technologies. 


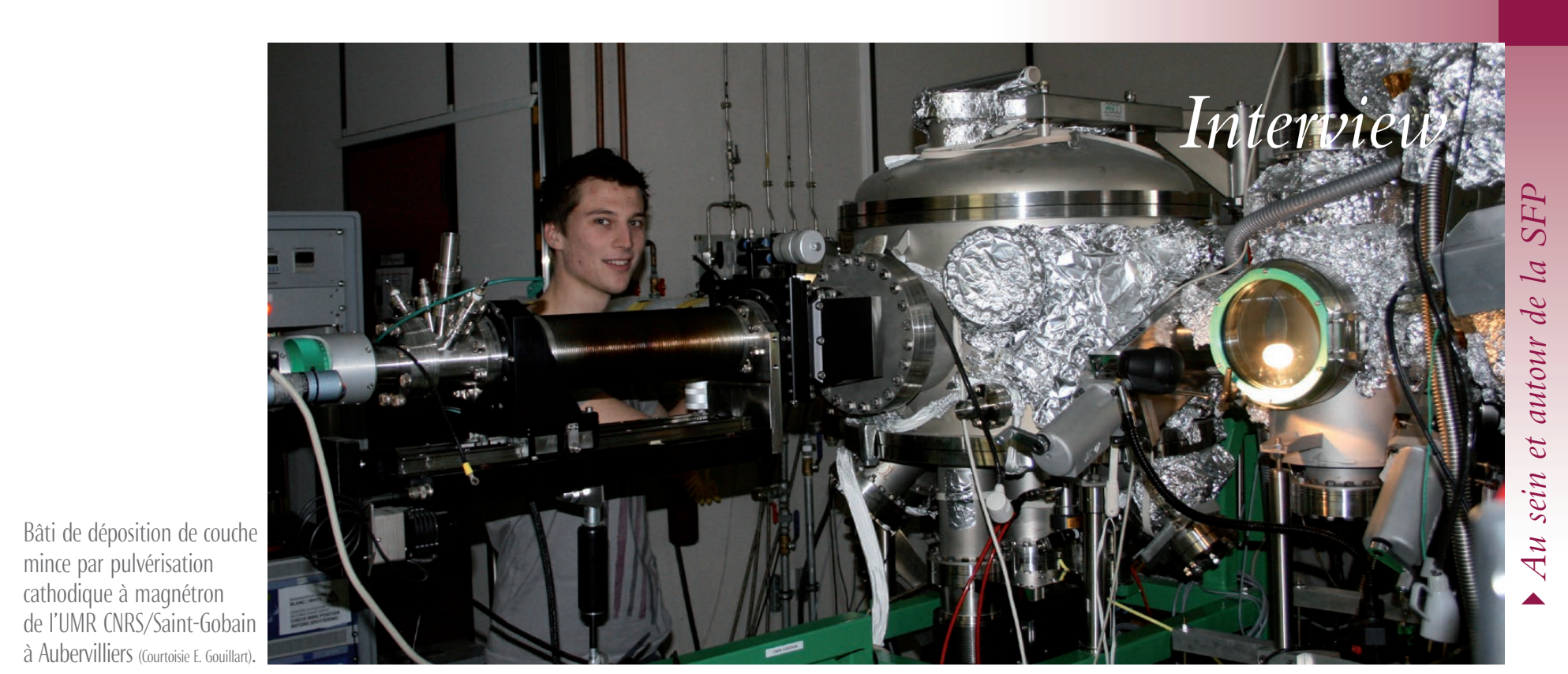

F\&P : Quels ont été vos mentors ou vos modèles lors de cette belle carrière? Quelle est votre recette pour réussir dans la direction des équipes?

CL : Mes mentors ont été des hommes qui croyaient en moi et m'ont offert des responsabilités toujours plus grandes. Je leur dois beaucoup... Je n'ai pas eu vraiment de modèles femmes à ces époques où je côtoyais essentiellement des hommes. J'ai progressé tout au long de ma carrière grâce aux personnes qui m'entouraient, en m'inspirant des bons exemples et en repérant les mauvais ! Je dis souvent que j'ai appris autant de mes équipes que de mes supérieurs..

Chaque leader a son style personnel et je ne suis pas sûre qu'il y ait «la " bonne recette pour diriger une équipe. Pour ma part, si je devais donner quelques ingrédients, ce serait :

- avoir une vision claire de son projet et la partager avec son équipe, - écouter les autres et les aider à s'épanouir,

- donner l'exemple en travaillant dur et en s'efforçant d'atteindre l'excellence,

- rester soi-même en connaissant ses points faibles et ses points forts, - aimer son travail et le faire avec plaisir.

F\&P : Quels sont vos liens avec la recherche académique? $\mathbf{C L}$ : J'ai fait des sciences dans l'industrie, mais toujours en lien étroit avec le monde académique, et dans un contexte de grande diversité. Saint-Gobain a plusieurs unités mixtes avec le CNRS, dont celle d'Aubervilliers dirigée par Emmanuelle Gouillart (interviewée dans Reflets de la physique, $\mathrm{n}^{\circ} 41$, p. 52). Les directeurs de la recherche de Saint-Gobain que j'ai connus ont toujours accordé une grande importance aux collaborations universitaires et à la formation par la recherche. À Aubervilliers, $80 \%$ de nos chercheurs ont une thèse. Dès la fin des années 70, J.P. Causse (le responsable scientifique de l'époque) avait incité les responsables de centres à embaucher des docteurs.

Saint-Gobain a également plusieurs Chaires en France (X-ESPCI, École des Ponts, INSA de Lyon, Mines de Nancy). J'ai moimême contribué à la mise en place d'un réseau international de partenaires académiques (Saint-Gobain University Network) dont l'ambition est double : identifier les meilleures équipes internationales pour collaborer avec elles, et utiliser ces partenariats pour recruter des étudiants talentueux après leur thèse dans les disciplines d'intérêt pour Saint-Gobain.
J'ai toujours beaucoup apprécié d'être en relation et d'échanger avec des équipes de différents continents et de différentes formations. La diversité est une grande richesse, porteuse d'innovation.

S\&P : Quelles sont vos motivations pour candidater à la viceprésidence de la SFP?

CL : Je ne suis pas docteure en physique, mais me suis toujours intéressée à la physique et à ses applications. Depuis près de quarante ans, j'ai interagi avec beaucoup de plaisir avec de nombreux physicien.ne.s. J'ai été incitée à accepter l'invitation cordiale de Michel Spiro par Didier Roux et Jean-Claude Lehmann, qui sont tous deux venus à Saint-Gobain après une carrière au CNRS et qui connaissent bien la complémentarité des deux milieux.

Il est encore un peu tôt pour moi pour développer ce que pourra être mon action à la SFP, mais je sais déjà que je vais pouvoir m'appuyer sur une équipe sympathique et solide avec laquelle je pourrai établir une feuille de route. Je compte m'inscrire dans la continuité de mes prédécesseurs et capitaliser sur les atouts reconnus de la société. De par mon parcours, je pense pouvoir renforcer les liens de la SFP avec l'industrie (y favoriser par exemple l'insertion de jeunes doctorants) et je m'attacherai tout particulièrement à poursuivre les actions menées pour attirer les jeunes, et notamment les jeunes filles, vers les carrières scientifiques (Olympiades, "Main à la pâte »...). Enfin, j'aurai à cœur de développer l'image et la visibilité de la SFP à l'extérieur et d'attirer de jeunes adhérents.

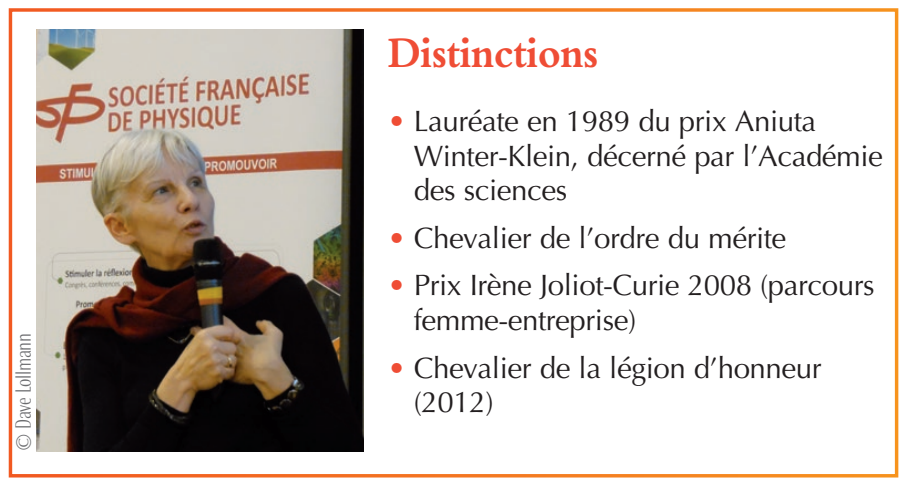

